\title{
Efeitos do consumo de bebida alcoólica sobre o feto
}

\author{
Effects of alcohol consumption during pregnancy
}

Tácio de Melo Freire ${ }^{1}$, José Cassiano Machado ${ }^{2}$, Enaldo Vieira de $\mathrm{Melo}^{3}$, Débora Gusmão Melo

\section{RESUMO}

Objetivo: verificar o consumo de bebida alcoólica durante a gravidez e avaliar os efeitos teratógenos do álcool no feto. Método: no período de abril a novembro de 2004, foram entrevistadas por meio do questionário T-ACE, 150 puérperas de uma maternidade de Ribeirão Preto/SP e dividas em consumidoras e não-consumidoras de álcool durante a gestação. Os recém-nascidos (RNs) destas mulheres foram avaliados em relação a peso, comprimento e perímetro cefálico ao nascimento. Estes parâmetros antropométricos foram comparados com o consumo de bebida alcoólica pelas mulheres. A análise dos dados foi realizada usando os testes $t$ de Student, teste de Mann-Whitney U e teste do $\chi^{2}$, sendo adotado nível de significância de 5\%. Resultados: das puérperas entrevistadas, 79,3\% (119) não foram identificadas como consumidoras de álcool pelo questionário T-ACE, ao passo que 20,7\% (31) foram consideradas consumidoras pelo mesmo instrumento. A média de comprimento dos RNs cujas mães não consumiram álcool foi de $48,2 \mathrm{~cm}$, ao passo que naqueles cujas mães ingeriram bebida alcoólica foi de $46,7 \mathrm{~cm}$ e esta diferença foi significante. Também se observou redução média de $109 \mathrm{~g}$ no peso e de $0,42 \mathrm{~cm}$ no perímetro cefálico nos fillhos de mães consumidoras de álcool, sendo que nos fetos do sexo feminino a redução de peso foi mais acentuada (186 g). Conclusão: o questionário T-ACE mostrou-se instrumento sensível para rastreamento do consumo alcoólico durante a gravidez. O uso de álcool na gravidez esteve associado à restrição do crescimento fetal, sendo os fetos do sexo feminino aparentemente mais suscetíveis aos efeitos do álcool.

PALAVRAS-CHAVE: Alcoolismo/diagnóstico; Feto/anormalidades; Transtornos relacionados ao uso do álcool; Consumo de bebidas alcoólicas/efeitos adversos; Complicações na gravidez

\section{ABSTRACT}

Purpose: to check alcohol consumption during pregnancy and to estimate teratogenic effects on the fetus. Methods: the TACE questionnaire was applied to 150 women who had just given birth in a maternity hospital in Ribeirão Preto/SP, from April to November 2004. The patients were divided into those who consumed and those did not consume alcohol during gestation. The newborns of these women were evaluated regarding weight, length and head circumference at birth. These anthropometric measurements were compared with the alcoholic beverage consumption by the women. The data analysis was performed by Student's $t$ test, Mann-Whitney U-test and $\chi^{2}$ test, with the level of significance set at $5 \%$. Results: among the interviewed women, $79.3 \%$ (119) were not identified as alcohol consumers by the T-ACE questionnaire and 20.7\% (31) were considered consumers by this questionnaire. The newborns whose mothers did not consume alcohol had a mean length of $48.2 \mathrm{~cm}$. On the other hand, those whose mothers used alcohol were $46.7 \mathrm{~cm}$ long, and such difference was statistically significant. A mean decrease of $109 \mathrm{~g}$ in weight and $0.42 \mathrm{~cm}$ in head circumference was observed in the children whose mothers had consumed alcohol. In the female fetuses the weight decrease was more pronounced (186 g). Conclusion: the T-ACE questionnaire was an effective screening instrument for detecting alcohol consumption during pregnancy. The use of alcohol during pregnancy was associated with fetal growth restriction. Apparently, the female fetuses were more susceptible to the effects of the use of alcohol during pregnancy.

KEYWORDS: Alcoholism/diagnosis; Fetus/abnormalities; Alcohol-related disorders; Alcohol drinking/adverse effects; Pregnancy complications

1 Acadêmico da Faculdade de Medicina do Centro Universitário Barão de Mauá - Ribeirão Preto (SP) - Brasil.

2 Docente de Ginecologia e Obstetrícia da Faculdade de Medicina do Centro Universitário Barão de Mauá - Ribeirão Preto (SP) - Brasil.

3 Médico do Hospital Santa Casa de Misericórdia de Ribeirão Preto (SP) - Brasil.

4 Professora de Genética Médica da Faculdade de Medicina do Centro Universitário Barão de Mauá - Ribeirão Preto (SP) - Brasil.

Faculdade de Medicina do Centro Universitário Barão de Mauá

Hospital Santa Casa de Misericórdia de Ribeirão Preto-SP

Correspondência: Débora Gusmão Melo

Av. Caramuru, 630, Bloco 01, apto. 401 - Bairro República - 14030-000 - Ribeirão Preto-SP - Telefones: 0 xx $163911-6719$ / 602-2598 / 602-3050 / 9991-3097 - Fax: (16) 633-0485 - e-mail: debora@rge.fmrp.usp.br

Recebido em: 4/3/2005 Aceito com modificações em: 9/8/2005

Rev Bras Ginecol Obstet. 2005;27(7): 376-81 


\section{Introdução}

Entre as substâncias conhecidas como teratógenas, o álcool é, provavelmente, o mais estudado. $\mathrm{Na}$ Inglaterra da primeira metade do século XVIII, quando o consumo de gim era disseminado, as crianças nascidas de mães etilistas foram descritas como fracas, débeis e desatentas. Mesmo assim, até o início do século XX a idéia de que beber durante a gravidez poderia prejudicar o desenvolvimento do feto era interpretada como moralista ${ }^{1}$.

Os efeitos teratógenos relacionados ao uso de álcool foram inicialmente descritos em 1968, mas só em 1973 foi definido um padrão específico de malformações nas crianças nascidas de mulheres etilistas, denominado de síndrome alcoólica fetal (SAF) ${ }^{2}$. A SAF é uma condição irreversivel caracterizada por anomalias craniofaciais típicas, deficiência de crescimento, disfunções do sistema nervoso central e várias malformações associadas $^{2}$. O termo "efeitos fetais do álcool" foi proposto para um grupo de crianças expostas ao álcool intra-útero, mas que não possuíam o quadro clínico completo de $\mathrm{SAF}^{3}$.

O álcool ingerido pela gestante atravessa a barreira placentária, o que faz com que o feto esteja exposto às mesmas concentrações do sangue materno. Porém, a exposição fetal é maior, devido ao metabolismo e eliminação serem mais lentos, fazendo com que o líquido amniótico permaneça impregnado de álcool não modificado (etanol) e acetaldeído (metabólito do etanol) ${ }^{4}$.

O etanol induz a formação de radicais livres de oxigênio que são capazes de danificar proteínas e lipídeos celulares, aumentando a apoptose e prejudicando a organogênese. Também inibe a síntese de ácido retinóico, que é uma substância reguladora do desenvolvimento embrionário ${ }^{5}$. Tanto o etanol, quanto o acetaldeído, têm efeitos diretos sobre vários fatores de crescimento celular, inibindo a proliferação de certos tecidos ${ }^{5}$.

Entretanto, a suscetibilidade fetal ao álcool é modulada por quantidade ingerida, época da exposição, estado nutricional e capacidade de metabolização materna e fetal ${ }^{6}$. A quantidade segura de álcool que uma gestante pode consumir não está definida na literatura, por isto recomenda-se abstinência total durante toda a gravidez. Estudos comprovam que o consumo de 20 gramas de álcool já é suficiente para provocar supressão da respiração e dos movimentos fetais, observados por meio de ultra-sonografia ${ }^{7}$.

Além disto, o consumo de álcool na gestação está relacionado ao aumento do número de abor- tos e a fatores comprometedores do parto, como risco de infecções, descolamento prematuro de placenta, hipertonia uterina, prematuridade do trabalho de parto e líquido amniótico meconial ${ }^{8}$.

A ocorrência de alcoolismo é subdiagnosticada durante a gestação, provavelmente pelo despreparo dos profissionais da saúde para investigar adequadamente ou valorizar as queixas compativeis com o hábito de beber ${ }^{9}$. Mensurar ingestão alcoólica não é fácil e vários instrumentos para rastreamento de abuso de álcool são conhecidos, como os questionários CAGE, MAST e T-ACE ${ }^{10}$. Estes questionários são destinados a caracterizar ou medir a quantidade, freqüência, intensidade e padrão de consumo de bebida alcoólica ${ }^{11}$. Comparando-se a eficácia do questionário T-ACE e da anamnese convencional (sem utilização de nenhum instrumento específico) na identificação do consumo de álcool entre 250 mulheres grávidas de Massachusetts, EUA, o questionário T-ACE demonstrou ser ótimo instrumento para rastreamento do uso de bebida alcoólica, detectando freqüência de consumo de $43 \%$ entre estas mulheres, ao passo que a anamnese convencional detectou apenas $9 \%$. Sua grande vantagem é a possibilidade de ser aplicado em dois minutos de conversa, sendo apropriado para rotina e prática nos serviços de obstetrícia e ginecologia ${ }^{12}$. Este questionário também se mostrou mais eficiente, com maior especificidade $(89 \%)$ e sensibilidade (69\%) que os questionários CAGE e MAST quando aplicados em gestantes ${ }^{10}$.

Calcula-se que $10 \%$ das crianças nascidas com restrição de crescimento intra-uterino nos EUA tenham como etiologia deste problema a exposição fetal ao álcool ${ }^{13}$, e ainda, que $11 \%$ dos pacientes institucionalizados por deficiência mental naquele país sofram dos efeitos teratógenos do álcool ${ }^{14}$. No Brasil existem poucos dados sobre a incidência de efeitos teratógenos do álcool. Estima-se a incidência de alcoolismo materno em 6/ 1.000 gestantes e a incidência de SAF em 1/ 1.000 recém-nascidos $(\mathrm{RNs})^{15}$. No entanto, calcula-se que um quarto das grávidas deste país faça uso esporádico de bebida alcoólica ${ }^{16}$.

O presente estudo teve como objetivo verificar o consumo de álcool na gravidez e avaliar os possiveis efeitos teratógenos desta substância sobre os fetos.

\section{Métodos}

A pesquisa foi realizada na maternidade do Hospital Santa Casa de Misericórdia de Ri- 
beirão Preto/SP, que atende mulheres pelo Sistema Único de Saúde (SUS), provenientes de todas as regiões da cidade e até de municípios próximos. O projeto de pesquisa foi autorizado pelo Comitê de Ética da instituição e a participação das mulheres foi mediante assinatura, após concordância com o termo de consentimento livre e esclarecido.

Participaram do estudo 150 puérperas internadas nesta maternidade, no periodo de abril a novembro de 2004. O instrumento empregado para mensurar o uso de bebida alcoólica durante a gravidez foi o questionário T-ACE, padronizado por Sokol et al. ${ }^{10}$ e modificado por Fabri ${ }^{11}$. Este questionário é composto por quatro questões principais que permitem avaliar tolerância ao álcool, existência de aborrecimento com relação às críticas de familiares e terceiros sobre o modo de beber da mulher, percepção da necessidade de redução do consumo de bebida alcoólica e persistência e dependência em relação à substância. Cada uma das quatro questões possui uma pontuação, que varia de 0 a 2 pontos para a primeira questão e de 0 a 1 ponto da segunda à quarta questões, estabelecendo-se pontuação total para o questionário que pode variar de 0 a 5 pontos. Pontuação total igual ou maior que 2 caracteriza a mãe como T-ACE positiva, ou seja, consumidora de no mínimo $28 \mathrm{~g}$ diárias de álcool absoluto. As questões do T-ACE são aplicadas intercaladamente com outras questões, que tratam de comportamento e hábitos alimentares durante a gestação, que não possuem nenhuma importância e não interferem no resultado do instrumento. São utilizadas para que mecanismos de defesa não sejam despertados e as mulheres não assumam postura de negação, omitindo informações fundamentais que alterariam os resultados do TACE.

Como critério para seleção das 150 puérperas escolheu-se sempre a última paciente a dar à luz no dia anterior à aplicação do questionário, desde que esta mulher fosse procedente do municipio de Ribeirão Preto. Na eventualidade da paciente escolhida ser proveniente de outro municipio, ou dela não aceitar participar da pesquisa, era selecionada a puérpera que deu a luz, imediatamente anterior, e assim subseqüentemente.

As mulheres foram distribuídas em dois grupos: T-ACE positivo e T-ACE negativo. Destas puérperas foram obtidos alguns dados de identificação como idade, estado civil e história obstétrica pregressa. As informações sobre as características das puérperas são apresentadas conforme a paridade, dividindo-as em primigestas e nãoprimigestas; conforme o estado civil dividindo-as em solteiras e casadas; e conforme a história positiva de aborto prévio ou não.

Os RNs destas 150 puérperas foram examinados clinicamente, registrando-se seus dados antropométricos (peso, comprimento e perimetro cefálico), sexo, Apgar no primeiro e quinto minuto de vida e Capurro somático.

O programa SPSS (versão 12.0) ${ }^{17}$ foi utilizado para as análises estatísticas. A descrição de todas as variáveis numéricas foi realizada pelo uso médias e desvios-padrão. Foi utilizado o teste $t$ de Student com dados independentes para as variáveis peso, comprimento dos RNs e Capurro somático, avaliando diferenças entre médias dos grupos de puérperas T-ACE positivo e T-ACE negativo. O teste de Mann-Whitney U foi utilizado para variáveis Apgar no primeiro e quinto minuto de vida, avaliando os mesmos grupos.

O teste do $\chi^{2}$ foi utilizado para avaliar associação entre estado civil e história prévia de aborto nos grupos de puérperas T-ACE positivo e T-ACE negativo.

\section{Resultados}

A idade das mulheres entrevistadas variou de 15 a 41 anos, com média de 23 anos e 6 meses e desvio-padrão (DP) de 5 anos e 4 meses. Setenta e seis mulheres eram casadas ou em união consensual $(50,7 \%)$ e as outras $49,3 \%$ (74) se declararam como solteiras. Quanto à paridade, 30\% (45) eram primíparas e 70\% (105) tinham gestações anteriores. Em relação a abortos, $71 \%$ (106) negaram perda fetal prévia.

Do total das 150 mulheres, 119 (79,3\%) foram consideradas tendo T-ACE negativo e 31 $(20,7 \%)$ T-ACE positivo. Destas 31 mulheres do grupo T-ACE positivo, 12 atingiram pontuação mínima igual a dois, 7 alcançaram três pontos, 10 alcançaram quatro pontos e apenas duas puérperas obtiveram a pontuação máxima do questionário T$\mathrm{ACE}$, cinco pontos.

O teste do $\chi^{2}$ mostrou que não há associação entre histórico de aborto prévio e o questionário T-ACE. Contudo, entre as 74 mulheres solteiras, o consumo de bebida alcoólica foi significantemente maior que nas casadas $\left(\chi^{2}=15,32 ; p=0,0001\right)$, como pode ser visto na Tabela 1 . 
Tabela 1 - Distribuição das 150 puérperas segundo o estado civil e o consumo de bebida alcoólica durante a gravidez.

\begin{tabular}{lcccccc}
\hline & \multicolumn{3}{c}{ Estado civil das puérperas } & \multicolumn{2}{c}{ Total } \\
T-ACE positivo** & \multicolumn{2}{c}{ solteiras } & \multicolumn{2}{c}{ casadas } & & \\
T-ACE negativo & $\mathbf{n}$ & $\%$ & \multicolumn{1}{c}{$\mathbf{n}$} & $\%$ & \multicolumn{1}{c}{$\mathbf{n}$} & $\%$ \\
\hline & 25 & 16,6 & 6 & 4,0 & $31^{*}$ & 20,7 \\
& 49 & 32,7 & 70 & 46,7 & 119 & 79,3 \\
& 74 & 49,3 & 76 & 50,7 & 150 & 100,0 \\
\hline
\end{tabular}

${ }^{*} p=0,001$.

**mais de dois pontos no questionário T-ACE

Entre os RNs, $83(55,6 \%)$ eram do sexo feminino e $67(44,4 \%)$ masculino. O comprimento médio destes RNs foi de $47,9 \mathrm{~cm}(\mathrm{DP}=2,9 \mathrm{~cm})$; o peso médio foi de $3.099 \mathrm{~g}(\mathrm{SD}=553 \mathrm{~g})$. A média de perímetro cefálico foi de $34,2 \mathrm{~cm}(\mathrm{DP}=1,7 \mathrm{~cm})$; e o Capurro somático médio foi de 38 semanas e 4 dias $(\mathrm{DP}=3$ dias). A maior parte (74) dos RNs obtiveram Apgar de 8 ou 9 no primeiro minuto de vida e de 10 no quinto minuto. Em relação a todas estas variáveis não houve diferença estatística entre os RNs do sexo feminino e masculino (comprimento $(p=0,72)$; peso $\mathrm{p}=0,15$ e perímetro cefálico $(\mathrm{p}=0,34))$, nem entre os RNs filhos de mães primigestas e não primigestas (comprimento $(p=0,72)$; peso $(p=0,14)$ e perímetro cefálico $(p=0,13))$.

Observou-se que os parâmetros antropométricos (peso, comprimento e perímetro cefálico) dos RNs cujas mães eram do grupo T-ACE positivo foram todos reduzidos, quando comparados aos RNs cujas mães eram do grupo T-ACE negativo (Figuras 1,2 e 3). Houve diminuição de $109 \mathrm{~g}$ no peso $(\mathrm{p}=0,97), 1,5 \mathrm{~cm}$ no comprimento $(\mathrm{p}=0,006)$ e 0,42 $\mathrm{cm}$ no perímetro cefálico $(\mathrm{p}=0,26)$ no grupo de RNs cujas mães foram do grupo T-ACE negativo. No entanto, apenas o comprimento apresentou redução significante pelo teste $t$ de Student $(\mathrm{p}<0,05)$.

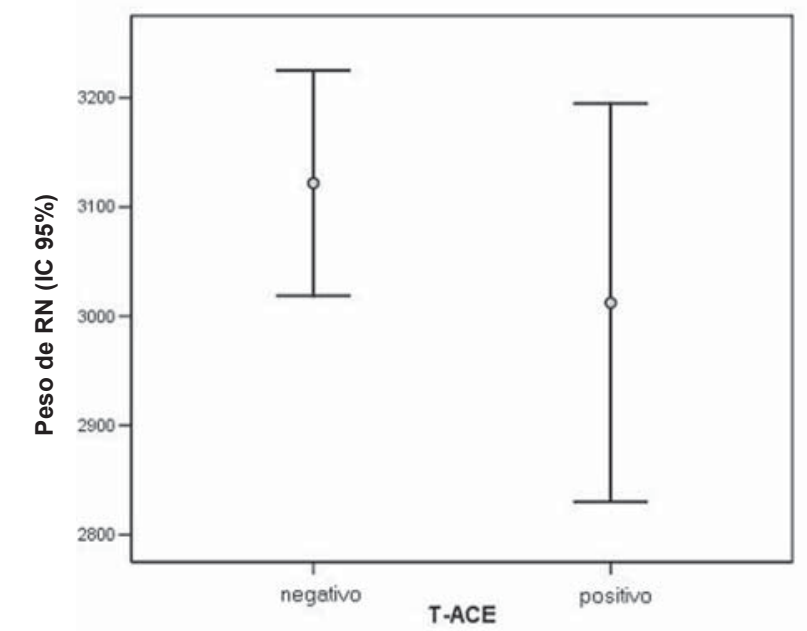

Figura 1 - Distribuição do peso dos RNs segundo o consumo de álcool na gravidez $(p=0,97)$.

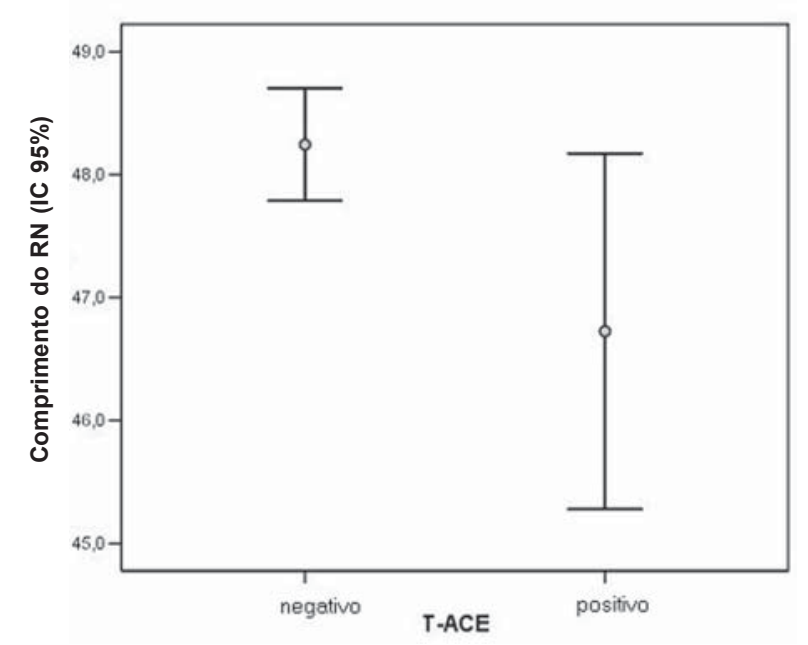

Figura 2 - Distribuição do comprimento dos RNs segundo o consumo de álcool na gravidez $(p=0,006)$.

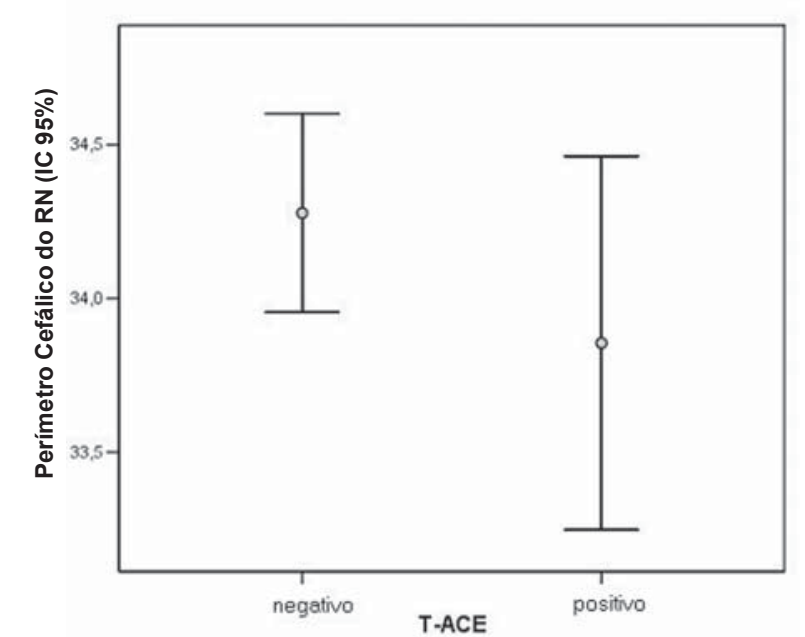

Figura 3 - Distribuição do perímetro cefálico dos RNs segundo o consumo de álcool na gravidez $(\mathrm{p}=0,26)$.

\section{Discussão}

O consumo de bebida alcoólica durante o primeiro trimestre de gravidez está comprovadamente associado ao aumento de risco de malformações fetais ${ }^{2}$. No presente estudo, entre as mulheres que tinham questionário T-ACE positivo não foi observado nenhum caso de anomalia fetal. No entanto, sabe-se que os efeitos teratógenos do álcool podem ocorrer na ausência de anomalias congênitas ou de $\mathrm{SAF}^{2}$.

Crianças expostas ao álcool no período prénatal mostram resultados piores nas escalas de desenvolvimento neuropsicomotor no primeiro ano de vida ${ }^{18}$. Filhos de mães que usaram álcool durante a gravidez nascem com peso, comprimento e perímetro cefálico menores. Além disto, persis- 
te velocidade de crescimento diminuída até os três anos de vida ${ }^{19}$. Um estudo longitudinal que acompanhou 500 crianças filhas de mães etilistas do nascimento até os 14 anos constatou que os efeitos teratógenos do álcool refletem-se no peso, comprimento e perímetro cefálico destas crianças e que os dados antropométricos podem ser sinalizadores dos efeitos neurocomportamentais relacionados à exposição pré-natal ao etanol ${ }^{20}$.

Comparando-se os efeitos do álcool, do cigarro, da cocaína e dos opiáceos sobre os parâmetros antropométricos de 417 RNs de etnia negra percebe-se que todas estas substâncias estão relacionadas com diminuição do peso, comprimento e perímetro cefálico ao nascimento. Entretanto, quando estes teratógenos são analisados separadamente o tabagismo resulta em diminuição média de $269 \mathrm{~g}$ no peso do RNs e o etilismo tem efeito mais devastador, reduzindo o peso em média de $509 \mathrm{~g}^{21}$.

Interessante notar que, embora sem significado estatístico, os dados do presente estudo apontam para maior sensibilidade aos efeitos fetais do álcool nos RNs do sexo feminino, pois nestas crianças a redução de peso foi mais acentuada, $186 \mathrm{~g}(\mathrm{p}=0,09)$. Resultados semelhantes são descritos em crianças com diagnóstico de SAF em North Dakota, EUA. Nesta amostra, 24,41\% dos RNs do sexo feminino apresentam peso ao nascer abaixo do terceiro percentil, ao passo que nos RNs do sexo masculino a freqüência de baixo peso é de $12,33 \%(\mathrm{p}<0,05)$. No entanto, a causa desta maior suscetibilidade nos RNs do sexo feminino em relação aos efeitos do álcoool permanece obscura ${ }^{22}$.

$\mathrm{Na}$ literatura, é relatada uma associação altamente significativa entre consumo de bebida alcoólica e gestantes solteiras ${ }^{23}$. Alguns estudos apontam para uma ocorrência duas vezes maior de consumo de bebida alcoólica durante a gravidez entre mulheres solteiras quando comparadas com casadas $^{24}$, possivelmente porque a gestação em mulheres solteiras com freqüência está associada a outros fatores de risco para o consumo de bebida alcoólica, como baixa escolaridade, baixo nível socioeconômico e gravidez indesejada ${ }^{24}$.

Há consenso entre os autores pesquisados que o uso de álcool durante a gravidez traz malefícios ao concepto. Um estudo com 445 puérperas de uma maternidade filantrópica da capital de São Paulo comprovou que $71,5 \%$ das mulheres têm algum conhecimento sobre os prejuízos do álcool ao bem-estar fetal e que $84,5 \%$ considera que nenhuma bebida alcoólica deve ser consumida durante a gravidez. Apesar disto, entre estas mulheres, apenas $48,8 \%$ afirmaram que foram orientadas a se abster do consumo alcoóli- co pelo profissional de saúde enquanto faziam prénatal ${ }^{9}$.

Percebe-se que os profissionais de saúde exercem importante papel na prevenção dos danos que o álcool pode causar aos fetos, devendo detectar precocemente as mulheres com historico de consumo abusivo ou não ${ }^{25}$.

Para evitar os efeitos teratógenos do álcool é necessário um trabalho profilático com as futuras mães, principalmente esclarecendo sobre as conseqüências que o etanol provoca no organismo fetal e que podem implicar dificuldades presentes e futuras. Para tanto, as informações devem causar impacto e ser capazes de induzir mudanças de comportamento.

\section{Referências}

1. Mattson SN, Riley EP. A review of the neurobehavioral deficits in children with fetal alcohol syndrome or prenatal exposure to alcohol. Alcohol Clin Exp Res. 1998;22(2):279-94.

2. Hoyme HE, May PA, Kalberg WO, Kodituwakku P, Gossage JP, Trujillo PM, et al. A practical approach to diagnosis of fetal alcohol spectrum disorders: clarification of the 1996 Institute of Medicine criteria. Pediatrics. 2005;115(1):39-47.

3. Hanson JW, Streissguth AP, Smith DW. The effects of moderate alcohol consumption during pregnancy on fetal growth and morphogenesis. J Pediatr. 1978;92(3):457-60.

4. Chaudhuri JD. Alcohol and developing fetus: a review. Med Sci Monit. 2000;6(5):1031-41.

5. Riley EP, Thomas JD, Goodlett CR, Klintsova AY, Greenough WT, Hungund BL, et al. Fetal alcohol effects: mechanisms and treatment. Alcohol Clin Exp Res. 2001;25(5 Suppl ISBRA):110S-116S.

6. Dawson DA, Das A, Faden VB, Bhaskar B, Krulewitch CJ, Wesley B. Screening for high- and moderaterisk drinking during pregnancy: a comparison of several TWEAK-based screeners. Alcohol Clin Exp Res. 2001;25(9):1342-9.

7. Kline J, Levin B, Stein Z, Susser M, Warburton D. Epidemiologic detection of low dose effects on developing fetus. Environ Health Perspect. 1981;42:119-26.

8. Parks KA, Nochajski TH, Wieczorek WF, Miller BA. Assessing alcohol problems in female DWI offenders. Alcohol Clin Exp Res. 1996;20(3):434-9.

9. Kaup ZOL, Merighi MAB, Tsunechiro MA. Avaliação do consumo de bebida alcoólica durante a gravidez. Rev Bras Ginecol Obstet. 2001;23(9):575-80. 
10. Sokol RJ, Martier SS, Ager JW. The T-ACE questions: practical prenatal detection of risk drinking. Am J Obstet Gynecol. 1989;160(4):863-8.

11.Fabri CE. Desenvolvimento e validação de instrumento para rastreamento do uso nocivo de álcool durante a gravidez (T-ACE) [dissertação]. Ribeirão Preto: Universidade de São Paulo; 2001.

12. Chang G, Wilkins-Haug L, Berman S, Goetz MA, Behr H, Hiley A. Alcohol use and pregnancy: improving identification. Obstet Gynecol. 1998;91(6):892-8.

13. Khoury MJ, Boyle C, De Coufle P, Floyd L, Hymbaughm K. The interface between dysmorphology and epidemiology in the diagnosis and surveillance for fetal alcohol effects. Pediatrics. 1996;98(2 Pt 1):315-6.

14. American Academy of Pediatrics. Committee on Substance Abuse and Committee on Children With Disabilities. Fetal alcohol syndrome and alcoholrelated neurodevelopmental disorders. Pediatrics. 2000;106(2 Pt 1):358-61.

15. Grinfeld H, Goldenberg S, Segre CA, Chadi G. Fetal alcohol syndrome in São Paulo, Brazil. Paediatr Perinatal Epidemiol. 1999;13(4):496-7.

16. Souza GT, Rodrigues MC, Ciavaglia MC. Análise do grau de conhecimento da populaçäo sobre a teratogenia do álcool e a conduta de enfermagem. Rev Bras Enfermagem. 1996;49(2):287-304.

17.SPSS for Windows, release 12.0 .0 [computer program]. Chicago: SPSS; 2003.
18. O'Connor MJ, Kasari C. Prenatal alcohol exposure and depressive features in children. Alcohol Clin Exp Res. 2000;24(7):1084-92.

19. Day NL, Robles N, Richardson G, Geva D, Taylor P, Scher M, et al. The effects of prenatal alcohol use on the growth of children at three years of age. Alcohol Clin Exp Res. 1991;15(1):67-71.

20.Sampson PD, Bookstein FL, Barr HM, Streissguth AP. Prenatal alcohol exposure, birthweight, and measures of child size from birth to age 14 years. Am J Public Health. 1994;84(9):1421-8.

21. Jacobson JL, Jacobson SW, Sokol RJ, Martier SS, Ager JW, Shankaran S. Effects of alcohol use, smoking and illicit drug use on fetal growth in black infants. J Pediatr. 1994;124(5 Pt 1):757-64.

22. Klug MG, Burd L, Martsolf JT, Ebertowski M. Body mass index in fetal alcohol syndrome. Neurotoxicol Teratol. 2003;25(6):689-96.

23. Burd L, Martsolf J, Klug MG, O’Connor E, Peterson M. Prenatal alcohol exposure assessment: multiple embedded measures in a prenatal questionnaire. Neurotoxicol Teratol. 2003;25(6):675-9.

24. Leonardson GR, Loudenburg R. Risk factors for alcohol use during pregnancy in a multistate area. Neurotoxicol Teratol. 2003;25(6):651-8.

25. Gupman AE, Svikis D, McCaul ME, Anderson J, Santora PB. Detection of alcohol and drug problems in an urban gynecology clinic. J Reprod Med. 2002;47(5):404-10. 